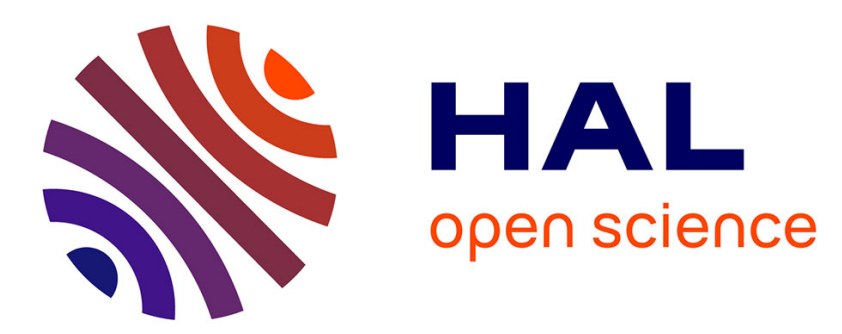

\title{
Pointwise characterization of the elastic properties of planar soft tissues: application to ascending thoracic aneurysms
}

Frances M. Davis, Yuanming Luo, Stéphane Avril, Ambroise Duprey, Jia Lu

\section{- To cite this version:}

Frances M. Davis, Yuanming Luo, Stéphane Avril, Ambroise Duprey, Jia Lu. Pointwise characterization of the elastic properties of planar soft tissues: application to ascending thoracic aneurysms. Biomechanics and Modeling in Mechanobiology, 2015, 14, pp.967-978. 10.1007/s10237-014-0646-9 . hal-01215247

\section{HAL Id: hal-01215247 \\ https://hal.science/hal-01215247}

Submitted on 14 Oct 2015

HAL is a multi-disciplinary open access archive for the deposit and dissemination of scientific research documents, whether they are published or not. The documents may come from teaching and research institutions in France or abroad, or from public or private research centers.
L'archive ouverte pluridisciplinaire HAL, est destinée au dépôt et à la diffusion de documents scientifiques de niveau recherche, publiés ou non, émanant des établissements d'enseignement et de recherche français ou étrangers, des laboratoires publics ou privés. 


\title{
Pointwise characterization of the elastic properties of planar soft tissues: Application to ascending thoracic aneurysms
}

\author{
Frances M. Davis · Yuanming Luo - Stéphane Avril - Ambroise \\ Duprey · Jia Lu
}

Received: date / Accepted: date

\begin{abstract}
In this manuscript, we present a combined experimental and computational technique that can identify the heterogeneous elastic properties of planar soft tissues. By combining inverse membrane analysis, digital image correlation, and bulge inflation tests, we are able to identify a tissue's mechanical properties locally. To show how the proposed method could be implemented, we quantified the heterogeneous material properties of a human ascending thoracic aortic aneurysm (ATAA). The ATAA was inflated at a constant rate using a bulge inflation device until it ruptured. Every $3 \mathrm{kPa}$ images were taken using a stereo digital image correlation system. From the images, the three dimensional displacement of the sample surface was determined. A deforming NURBS mesh was derived from the displacement data and the local strains were computed. The wall stresses at each pressure increment were determined using inverse membrane analysis. The local material properties of the ATAA were then identified using the pointwise stress and strain data. To show that it is necessary to consider the heterogeneous distribution of the mechanical properties in the ATAA, three different forward finite element simulations using pointwise, elementwise, and homogeneous material properties were compared. The forward finite element predictions revealed that heterogeneous nature of the ATAA must be
\end{abstract}

F. M. Davis · S. Avril · A. Duprey

Ecole Nationale Suprieure des Mines de Saint-Etienne

CIS-EMSE,CNRS:UMR5307,LGF

158 Cours Fauriel

42100 St. Étienne France

Y. Luo · J. Lu (Corresponding author)

Department of Mechanical and Industrial Engineering

The University of Iowa

Iowa City, IA 52242, USA

E-mail: jia-lu@uiowa.edu accounted for to accurately reproduce the stress-strain response.

Keywords bulge inflation test - heterogeneous material properties $\cdot$ digital image correlation $\cdot$ inverse elastostatic analysis $\cdot$ thoracic aneurysm

\section{Introduction}

Most soft biological tissues are heterogeneous, nonlinear, and anisotropic. Yet, these heterogeneous tissues are often modeled as homogeneous. For some tissues the assumption of homogeneity is justified but, frequently the assumption of homogeneity is simply convenient. The identification of the heterogeneous material properties can be particularly important when attempting to understand the interaction between disease progression or tissue repair and a tissue's mechanical properties. In, for example, aneurysm rupture and growth, the identification of a tissue's heterogeneous properties is essential. In aortic aneurysms, spatial variations in the mechanical properties occur naturally due to local remodeling of the vessel wall (Humphrey et al., 2014; Wilson et al., 2012). This regional variation in the mechanical behavior has a direct effect on the wall stress, which is the most popular biomechanical factor for assessing aneurysm rupture risk (Vorp, 2007). Furthermore, studying how the spatial distribution of the mechanical properties evolves as the aneurysm geometry and composition change could provide significant insight into the growth and remodeling process. Although the heterogeneous nature of aortic aneurysms is recognized, only a few investigators have characterized the spatial variation in mechanical properties (Choudhury et al., 2009; Haskett et al., 2010; Iliopoulos et al., 2009; Ní Annaidh et al., 2012; Romo et al., 2014). 
The primary barrier to investigating the heterogeneous nature of soft tissues is the difficulty developing experimental protocols that can accurately capture the spatial variation in mechanical properties. Conventional testing approaches, such as uniaxial and biaxial tensile tests, rely on the assumption of uniformity of the stress and strain in the center of the specimen. The most popular strategy for studying heterogeneity in soft tissues involves dividing the tissue into multiple test samples and characterizing the homogeneous response of each sample using uniaxial or biaxial tests. With this approach the regional heterogeneity of a tissue can be studied at a spatial resolution of centimeters. Using uniaxial or biaxial tests to study spatial variation in the material properties has two significant limitations: It requires the heterogeneity to be rather large sized and a significant number of samples.

The objective of this manuscript is to advance our ability to characterize heterogeneities in soft tissues in vitro. With this in mind, we restrict ourselves to planar soft tissues that can be modeled as membranes. From the mechanics perspective, membranes are thin structures that offer little resistance to bending. Many soft tissues can be modeled as membranes such as aneurysms, the bladder, skin, and the sclera in the eye (Humphrey, 2003; Myers et al., 2010; Tonge et al., 2013). Recently, there have been significant computational and theoretical developments in the formulation of inverse approaches to describe the finite deformation of membranes (Lu and Zhao, 2009; Lu et al., 2008; Zhao et al., 2009). To characterize the heterogeneous distribution of material properties in planar biological tissues we exploit these developments and the unique properties of membranes.

There are three primary components to the material characterization: bulge inflation tests, stereo digital image correlation (DIC), and inverse membrane analysis. By coupling bulge inflation tests with DIC to obtain full-field displacement measurements, we are able to compute the strain locally, as opposed to globally with traditional experimental protocols. The inverse membrane formulation capitalizes on a unique property of the membrane equilibrium equations, static determinacy, that allows the stress field to be identified without prior knowledge of the membrane's material properties (Genovese et al., 2014; Lu et al., 2008; Romo et al., 2014). A straight-forward pointwise characterization of a membrane's elastic properties can then be completed using the local stress-strain data from the inverse membrane analysis and the DIC measurements (Genovese et al., 2014; Lu and Zhao, 2009; Zhao et al., 2009, 2011a,b).
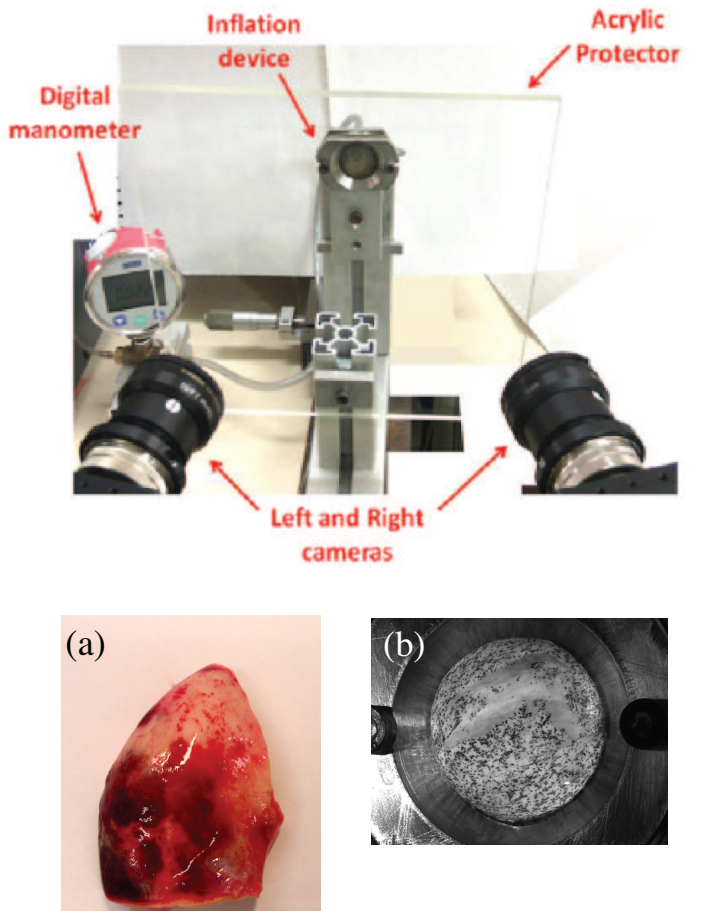

Fig. 1 Experimental setup and test sample (a) before testing and (b) after rupture

In this manuscript, we describe a protocol for the pointwise identification of anisotropic material properties in planar soft tissues. The method is applied to a human ascending thoracic aortic aneurysm (ATAA). The ATAA was subjected to a bulge inflation test and the displacement and pressure data collected are used as inputs to the inverse membrane method. The wall stresses were calculated using an inverse membrane formulation with a NURBS representation (Hughes et al., 2005; Lu et al., 2008). Using the computed full field stress-strain data, the pointwise distribution of the mechanical properties for the ATAA specimens were identified. To substantiate the claim that a pointwise resolution is necessary to characterize the mechanical properties of the ATAA a forward finite element analysis was performed using homogeneous, elementwise, and pointwise distributions of the mechanical properties and the resulting errors in the predicted displacement, strain, and stress are reported.

\section{Methods}

\subsection{Experimental Protocol}

An unruptured ATAA section was collected from a patient undergoing elective surgery to replace his ATAA with a graft in accordance with a protocol approved by the Institutional Review Board of the University Hospi- 
tal Center of St. Etienne. After retrieval, the specimen was placed in saline solution and stored at $4^{\circ} \mathrm{C}$ until testing, which occurred within 48 hours of the surgery. Immediately prior to testing, the ATAA was cut into a square specimen approximately 45 x $45 \mathrm{~mm}$. Any fatty deposits were removed from the surface of the tissue to ensure that during mechanical testing the tissue did not slip in the clamps. An average thickness was found for the sample by measuring the thickness of the tissue at a minimum of 5 locations.

The specimen was clamped in the bulge inflation device, Fig. 1, so that the luminal side of the tissue faced outward. Then a speckle pattern was applied to the luminal surface using black spray paint. The sample was inflated using a syringe pump driven at $2 \mathrm{~mL} / \mathrm{min}$ to infuse water into the cavity behind the sample. During the test, the pressure was measured using a digital manometer (WIKA, DG-10). Images of the inflating specimen were collected using a commercial DIC system (GOM, 5M LT) composed of two 8-bit CCD cameras equipped with $50 \mathrm{~mm}$ lenses (resolution: 1624 $\mathrm{x} 1236 \mathrm{px}$ ). The cameras were positioned $50 \mathrm{~cm}$ apart at an angle of $30^{\circ}$ with an aperture of $f / 11$. This produced a depth of field of $15.4 \mathrm{~mm}$ which was sufficient to capture the deformation of the tissue up to failure. Images of the deforming sample were collected every $3 \mathrm{kPa}$ until the sample ruptured.

After rupture, the collected images were analyzed using the commercial correlation software ARAMIS (GOM, v. 6.2.0) to determine the three dimensional displacement of the tissue surface. For the image analysis, a facet size of $21 \mathrm{px}$ and a facet step of $5 \mathrm{px}$ were chosen based on the speckle pattern dot size, distribution, and contrast. The selected parameters produced a cloud of approximately 15,000 points where the three displacement values were calculated.

A preliminary study was performed to estimate the displacement and strain noise floor for the DIC measurements. Four samples were inflated to 0, 3, 15, 30, 45 , and $60 \mathrm{kPa}$ and a baseline stage was captured and 1 s later another stage was captured under the same loading conditions. The baseline image was correlated with the second stage to determine the displacement at every point $i$. The error, $l$, was calculated at each image point (Myers et al., 2010)

$l_{i}=\sqrt{\left(r_{i}^{x}-R_{i}^{x}\right)^{2}+\left(r_{i}^{y}-R_{i}^{y}\right)^{2}+\left(r_{i}^{z}-R_{i}^{z}\right)^{2}}$

where $r_{i}^{x}, r_{i}^{y}$, and $r_{i}^{z}$ are the components of displacement in the second image and $R_{i}^{x}, R_{i}^{y}$, and $R_{i}^{z}$ are the components of displacement in the baseline image. The mean displacement error for a typical aortic specimen was $5 \mu \mathrm{m}$, with a maximum error that was always less than $30 \mu \mathrm{m}$. To estimate the lowest strain error possible for the DIC configuration, the mean value of the strain was calculated for the $0 \mathrm{kPa}$ case (Reu, 2014). The strain floor was found to be $0.6 \%$.

\subsection{Geometric Reconstruction}

A deforming NURBS mesh was extracted by morphing a NURBS template to the DIC point clouds. The template was a circular domain with a diameter slightly less than that of the point cloud in the first pressure state. The NURBS surface was parameterized as a single patch containing clamped knots of 20 divisions in each parametric direction, with $22 \times 22$ control points. Since NURBS control points, in general, do not fall on the surface they describe, they cannot be directly derived from the DIC clouds. Instead, the positions of the Gauss points were obtained first using a moving least-square method (Belytschko et al., 1996). For each Gauss point, a set of nearest image points in the DIC point cloud were identified based on their distance to the Gauss point in the first pressure state. The radius of the neighboring region was automatically adjusted so that it contained at least 6 image points. The position of each Gauss point, $\mathbf{y}_{g}$, was computed using an affine interpolation

$\mathbf{y}_{g}=\frac{\sum_{\mathbf{y} \in \Omega_{g}} w_{j} \mathbf{y}_{j}}{\sum_{\mathbf{y} \in \Omega_{g}} w_{j}}$

where $\mathbf{y}_{j}$ is the position vector for each image point in the neighborhood, $\Omega_{g}$, and $w_{i}$ is the weighting function taken to be the inverse of the distance from $\mathbf{y}_{j}$ to the Gauss point. Using the same weights calculated in the first stage, the Gauss points in every pressure stage were identified.

A global least squares problem was then formulated to compute the best-fit positions of the control points. The NURBS surface was represented as

$\mathbf{x}=\sum_{i} N_{i}\left(u_{1}, u_{2}\right) \mathbf{Q}_{i}$

where $N_{i}$ are the NURBS basis functions, $\mathbf{Q}_{i}$ are the control points, and the pair of knot variables, $\left(u_{1}, u_{2}\right)$, represent a material point. The position of a modeled Gauss point is then given by $\mathbf{x}_{g}=\sum_{i} N_{i}\left(u_{1 g}, u_{2 g}\right) \mathbf{Q}_{i}$. The control points were obtained by minimizing a weighted sum of $\left\|\mathbf{x}_{g}-\mathbf{y}_{g}\right\|^{2}$ over all Gauss points. This procedure was applied to each pressure state.

To assess the accuracy of the constructed NURBS mesh the distance deviation between the NURBS surface and the DIC point cloud was calculated. For each image point, $\mathbf{y}_{i}$, its closest projection point on the NURBS 
surface, $\mathbf{x}_{i}$, was computed (Lu, 2011). Points whose closest projection laid outside the NURBS domain were discarded. Using the remaining points the distance deviation was calculated

$d=\left(\frac{\sum_{i}\left\|\mathbf{x}_{i}-\mathbf{y}_{i}\right\|^{2}}{n}\right)^{1 / 2}$

where $n$ is the number of DIC cloud points inside the NURBS domain.

\subsection{Data Analysis}

Strain Surface strains were computed with the aid of the NURBS curvilinear coordinate system. The surface coordinates, $u_{\alpha},(\alpha, \beta=1,2)$ induce a set of convected basis vectors $\left(\mathbf{a}_{1}, \mathbf{a}_{2}\right)$ where $\mathbf{a}_{\alpha}=\frac{\partial \mathbf{x}}{\partial \mathbf{u}_{\alpha}}$ and $\mathbf{x}\left(u_{1}, u_{2}\right)$ is the NURBS representation given in Eq. 3. The reciprocal basis $\left(\mathbf{a}^{1}, \mathbf{a}^{2}\right)$ are computed such that $\mathbf{a}_{\alpha} \cdot \mathbf{a}^{\beta}=\delta_{\alpha}^{\beta}$. In the reference configuration, the basis vectors are denoted by $\left(\mathbf{A}_{1}, \mathbf{A}_{2}\right)$ and $\left(\mathbf{A}^{1}, \mathbf{A}^{2}\right)$.

The surface deformation gradient tensor is

$\mathbf{F}=\mathbf{a}_{\alpha} \otimes \mathbf{A}^{\alpha}$

It then follows that the surface Cauchy-Green deformation tensor, $\mathbf{C}$, and the Green-Lagrangian strain tensor, $\mathbf{E}$, are given by

$\mathbf{C}=\left(\mathbf{a}_{\alpha} \cdot \mathbf{a}_{\beta}\right) \mathbf{A}^{\alpha} \otimes \mathbf{A}^{\beta}$

$\mathbf{E}=\frac{1}{2}\left(\mathbf{a}_{\alpha} \cdot \mathbf{a}_{\beta}-\mathbf{A}_{\alpha} \cdot \mathbf{A}_{\beta}\right) \mathbf{A}^{\alpha} \otimes \mathbf{A}^{\beta}$.

The physical components of $\mathbf{C}$ and $\mathbf{E}$ are computed by constructing a local orthonormal basis $\left(\mathbf{G}_{1}, \mathbf{G}_{2}\right)$ in the tangent plane spanned by $\left(\mathbf{A}_{1}, \mathbf{A}_{2}\right)$. The physical components of the Cauchy-Green deformation tensor, $C_{i j}$, and Green-Lagrangian strain tensor, $E_{i j}$, are $C_{i j}=$ $\mathbf{G}_{i} \cdot \mathbf{C} \mathbf{G}_{j}$ and $E_{i j}=\mathbf{G}_{i} \cdot \mathbf{E} \mathbf{G}_{j}$, respectively.

Wall Stress For an inverse membrane boundary value problem the deformed configurations and boundary conditions are given as inputs to the finite element model and the wall stress is calculated. The balance equation that governs static equilibrium is (Green and Adkins, 1970; Lu et al., 2008)

$\frac{1}{\sqrt{a}}\left(\sqrt{a} t^{\alpha \beta} \mathbf{a}_{\alpha}\right)_{, \beta}+p \mathbf{n}=0$

where $a$ is $\operatorname{det}\left[\mathbf{a}_{\alpha} \cdot \mathbf{a}_{\beta}\right], \mathbf{t}$ is the Cauchy wall tension, $p$ is the applied internal pressure, $\mathbf{n}$ is an outward facing unit normal, and ()$_{, \beta}$ indicates $\frac{\partial}{\partial u^{\beta}}$. Note that the Cauchy wall tension $\mathbf{t}$ is directly related to the Cauchy stress, $\boldsymbol{\sigma}$, through the current thickness of the membrane, $h$, via $t^{\alpha \beta}=h \sigma^{\alpha \beta}=t^{\beta \alpha}$. If the thickness distribution is known for every element (or indeed every Gauss point) in the mesh the local Cauchy stress can be ascertained. Using our current experimental method the thickness distribution of the tissue cannot be measured accurately. To avoid introducing errors into the stress calculation due to imprecise thickness values we have elected to use the wall tension to describe the stress state of the tissue.

The weak form of the boundary value problem reads

$$
\int_{\Omega} t^{\alpha \beta} \mathbf{a}_{\alpha} \cdot \delta \mathbf{x}_{, \beta} d a-\int_{\Omega} p \mathbf{n} \cdot \delta \mathbf{x} d a=0
$$

where $\delta \mathbf{x}$ is any admissible variation to the current configuration $\Omega$. The details of the finite element procedure for solving Eq. 9 were presented in Lu et al. (2008). Briefly, the Cauchy wall tension is regarded as a function of the inverse deformation gradient. The weak form subsequently yields a set of nonlinear algebraic equations for the positions of control points in the reference configuration. While solving for the reference configuration the tension field in the current state is determined. An auxiliary material model is needed to perform the inverse analysis. The material model influences the predicted undeformed configuration; however, due to the static determinacy of Eq. 8, the influence on the wall tension is expected to be weak ( $\mathrm{Lu}$ et al., 2008, 2013; Miller and Lu, 2013). As in a previous study (Genovese et al., 2014), a neo-Hookean model was implemented. For computational efficiency, the stiffness parameter of the model was set to unrealistically high values to ensure a robust convergence.

To facilitate the inverse analysis, the control points on the outermost edge of the mesh were fixed. Since the DIC point cloud does not include the edge where the specimen was fixed to the clamp, the applied boundary conditions are only approximate. Applying any displacement based constraint in the inverse membrane analysis creates a boundary region in the solution where the stresses are inaccurate ( $\mathrm{Lu}$ et al., 2008). To minimize the influence of boundary effect, the outer ring of elements were deemed to be the boundary region and excluded from further analyses. By a retrospective comparison with the forward analysis reported later (Sec. 3.4) the size of the boundary region was confirmed.

Material Property Identification Using inverse membrane analysis, the stress was calculated at every Gauss point. Combining the stress data with the local surface strains calculated from Eqs. 5 - 7, the stress-strain response 
at every Gauss point in the mesh is known. The local material properties at each Gauss point were then identified by fitting the local stress-strain response to a hyperelastic surface energy density. A two-dimensional version of the strain energy density proposed by Gasser, Ogden, and Holzapfel (GOH) (2006) was used:

$w=\frac{\mu_{1}}{2}\left(\mathrm{I}_{1}-\ln \left(\mathrm{I}_{2}\right)-2\right)+\frac{\mu_{2}}{4 \gamma}\left(\mathrm{e}^{\gamma\left(\mathrm{I}_{k}-1\right)^{2}}-1\right)$

where $\mathrm{I}_{1}=\operatorname{tr} \mathbf{C}$ and $\mathrm{I}_{2}=\operatorname{det} \mathbf{C}$ are the principal invariants of the Cauchy-Green deformation tensor and $\mathrm{I}_{\kappa}=\mathbf{C}:(\kappa \mathbf{I}+(1-2 \kappa) \mathbf{M} \otimes \mathbf{M})$ is a compound invariant consisting of isotropic and anisotropic contributions. In the compound invariant $\mathrm{I}_{\kappa}$, the unit vector $\mathbf{M}=$ $\cos \theta \mathbf{G}_{1}+\sin \theta \mathbf{G}_{2}$ defines the preferred orientation of the collagen fibers in the reference configuration while $\kappa$ characterizes the dispersion of the collagen fibers.

The dispersion parameter, $\kappa$, varies from 0 to $\frac{1}{2}$. When $\kappa=0$ all of the collagen fibers are perfectly aligned in the direction of $\mathbf{M}$ and when the distribution of collagen fibers has no preferential direction (isotropic), $\kappa=\frac{1}{2}$. In Eq. 10, the parameters $\mu_{1}$ and $\mu_{2}$ are the effective stiffnesses of the matrix and collagen fibers, respectively, both having dimensions of force per unit length. The parameter $\gamma$ is a non-dimensional parameter that governs the tissue's strain stiffening response.

The second Piola-Kirchhoff wall tension, $\mathbf{S}$, is written as

$$
\begin{aligned}
\mathbf{S}=2 \frac{\partial w}{\partial \mathrm{I}_{1}} \mathbf{1}+2 & \frac{\partial w}{\partial \mathrm{I}_{2}} \mathrm{I}_{2} \mathbf{C}^{-1} \\
& +2 \frac{\partial w}{\partial \mathrm{I}_{\kappa}}(\kappa \mathbf{I}+(1-2 \kappa) \mathbf{M} \otimes \mathbf{M}) .
\end{aligned}
$$

Substituting Eq. 10 into Eq. 11 one finds

$$
\begin{aligned}
\mathbf{S} & =\mu_{1}\left(\mathbf{I}-\mathbf{C}^{-1}\right) \\
& +\mu_{2} \mathrm{e}^{\gamma\left(\mathrm{I}_{\kappa}-1\right)^{2}}\left(\mathrm{I}_{\kappa}-1\right)(\kappa \mathbf{I}+(1-2 \kappa) \mathbf{M} \otimes \mathbf{M})
\end{aligned}
$$

noting that the second Piola-Kirchoff wall tension is related to the Cauchy wall tension via $\mathbf{t}=\frac{1}{\sqrt{\mathrm{I}_{2}}} \mathbf{F} \mathbf{S ~ F}^{\mathrm{T}}$.

The values of the model parameters $\mu_{1}, \mu_{2}, \gamma, \kappa$, and $\theta$ were determined by minimizing the sum of the squares difference between the stress computed from the inverse membrane analysis and those computed using Eq. 12. The nonlinear minimization was solved in MATLAB (MathWorks, v. 7.14) where the model parameters were constrained such that: $\mu_{1}, \mu_{2}, \gamma>0$, $0 \leq \theta \leq \pi$, and $0 \leq \kappa \leq \frac{1}{2}$. To ensure that the minimization routine converged to a global minimum, three sets of initial guesses were used. Since the parameters $\theta$ and $\kappa$ are tightly bound, the upper bound, lower bound, and middle value were used. For the parameters $\mu_{1}, \mu_{2}$,

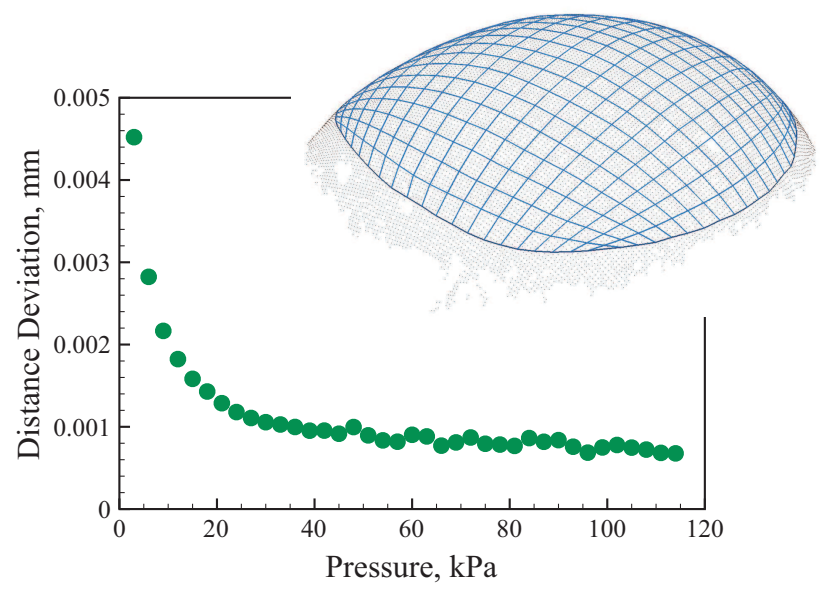

Fig. 2 NURBS mesh (blue) superimposed on the experimental DIC point cloud at a pressure of $117 \mathrm{kPa}$ along with a plot of the distance deviation calculated using Eq. 4 as a function of pressure.

and $\gamma$ random values between 0.01 and 5 were generated. The parameter identification was relatively unaffected by changes to the initial guess and consistently converged to the same solution.

Forward Validation The pointwise wall tensions and property distributions were validated using forward finite element analysis. This was accomplished by mapping the pointwise mechanical properties back to the NURBS mesh and simulating the inflation process. The configuration at a pressure of $3 \mathrm{kPa}$ was taken as the reference configuration. The applied pressure was assumed to balance the weight of the tissue in this configuration rendering it stress-free. Since the material parameters in the perimeter ring of elements were not identified, these elements were excluded from the forward analysis. Displacement boundary conditions, derived from the experimental DIC data, were applied to these elements. The wall tensions, strains, and displacements calculated in the forward analysis were compared to the DIC displacements, the DIC strains computed on the NURBS mesh, and the stresses from the inverse analysis. The relative error was computed at every Gauss point and averaged over the computational domain. To illustrate the influence of assuming pointwise heterogeneous properties, two additional forward validations were performed using an elementwise and a homogeneous distribution of mechanical properties. To identify the elementwise material properties the nonlinear minimization described earlier was applied to each of the interior elements. For the homogeneous case, all of the pointwise material properties were averaged to identify one set of material properties. The forward validations for the elementwise and the homogeneous cases 


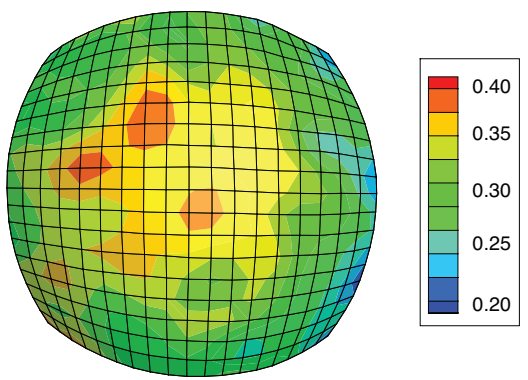

(a) Wall Tension $(\mathrm{N} / \mathrm{mm})$ at $30 \mathrm{kPa}$

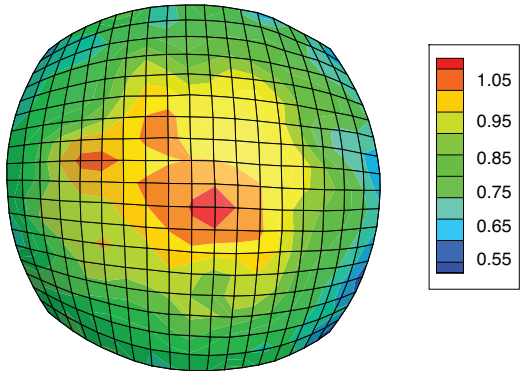

(c) Wall Tension $(\mathrm{N} / \mathrm{mm})$ at $75 \mathrm{kPa}$

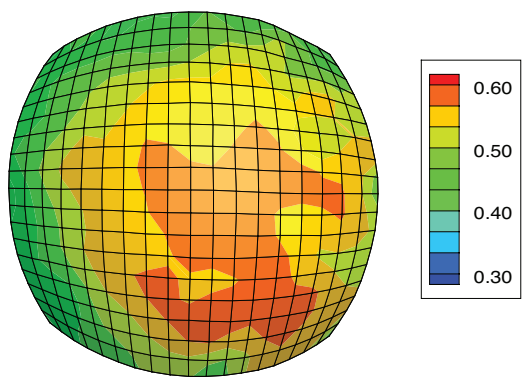

(b) Green-Lagrange strain at $30 \mathrm{kPa}$

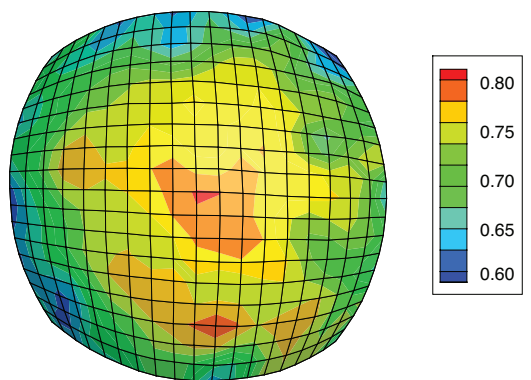

(d) Green-Lagrange strain at $75 \mathrm{kPa}$

Fig. 3 Contours of the magnitude of the wall tension (N/mm) and Green-Lagrange strain at (a,b) $30 \mathrm{kPa}$ and (c,d) $75 \mathrm{kPa}$.

were performed using the same method described for the pointwise case.

\section{Results}

A bulge inflation test to failure was performed on an ATAA collected from a male patient who was 55 years old. The diameter of the aneurysm as determined by pre-surgical CT scan was $55 \mathrm{~mm}$. The sample ruptured at $117 \mathrm{kPa}$. However, it must be noted that, in terms of the stress it induces the pressure applied in the test is not equivalent to a blood pressure, for example, $15 \mathrm{kPa}$ measured by the pressure sensor is not equivalent to a blood pressure of $15 \mathrm{kPa}(113 \mathrm{mmHg})$ due to the new shape of the specimen. The pressure and DIC data collected during the bulge inflation test were used to generate a deforming NURBS mesh and identify the local stress-strain response during the inflation test. Using the pointwise stress-strain data, the spatial distribution of the mechanical properties was identified.

\subsection{Geometric Reconstruction}

Using the experimental DIC point cloud a deforming NURBS mesh was generated of the ATAA sample. Figure 2 shows the extracted NURBS mesh at a pressure of $117 \mathrm{kPa}$ superimposed on the experimental DIC point cloud at the same pressure. Using Eq. 4 the distance deviation for each pressure stage was computed. As shown in Fig. 2, the overall the distance deviations are very small, however the values are significantly larger at low pressures. The distance deviation rapidly decreases as the tissue is inflated and stabilizes by $30 \mathrm{kPa}$.

\subsection{Local Stress and Strain Response}

Figure 3 shows the distributions of the magnitude of the Cauchy wall tension, $\overline{\mathbf{t}}$, and Green-Lagrangian strain, $\overline{\mathbf{E}}$, at an applied pressure of $30 \mathrm{kPa}$ (Fig. 3a,b) and $75 \mathrm{kPa}$ (Fig. 3c,d). The distribution of wall tension and strain remained similar throughout the inflation of the specimen. Components of the Cauchy wall tension and Green Lagrange strain at the center of each element in the computational domain are provided in an on-line supplementary data file.

In general, at each Gauss point both the normal strains and the planar shear strains were non-zero. To facilitate plotting of the local stress-strain response, the axes of principal strain were identified and the local stresses and strains were rotated into the principal strain axes. In Fig. 4, the three components of the wall tension in the principal strain axes, $\tilde{t}_{11}, \tilde{t}_{12}$, and $\tilde{t}_{22}$ are plotted against the principal stretches $\lambda_{1}$ and $\lambda_{2}$. As expected, the local stress-strain response shows the non-linear stiffening behavior that is common in arteries. In a small region where rupture eventually occurred the ATAA appears to yield (Fig. 4c). The locations of 
Table 1 Mean value and standard deviation of the material parameters.

\begin{tabular}{|c|c|}
\hline Material Parameters & Mean \pm Std \\
\hline$\mu_{1}(\mathrm{~N} / \mathrm{mm})$ & $0.0122 \pm 0.0111$ \\
\hline$\mu_{2}(\mathrm{~N} / \mathrm{mm})$ & $0.2747 \pm 0.1051$ \\
\hline$\gamma$ & $1.6090 \pm 0.3527$ \\
\hline$\kappa$ & $0.4019 \pm 0.0478$ \\
\hline$\theta(\mathrm{rad})$ & $1.5560 \pm 0.6003$ \\
\hline
\end{tabular}

this localized yielding correspond to strain concentrations in zone where rupture initiates (Fig. 7c).

\subsection{Material Property Identification}

The proposed model for the elastic behavior of the ATAA was able to fit the bulge inflation data well $(0.81<$ $\left.\mathrm{R}^{2}<0.99\right)$. Lower values of the correlation coefficient were located in the small zone where rupture eventually occurred. Excluding this region the minimum value of $\mathrm{R}^{2}$ was 0.96 . The experimental data (points) and model fits (lines) for three Gauss points are shown in Fig. 4.

The distributions of the material parameters are plotted in Fig. 5 and they clearly display a heterogeneous distribution. The parameter $\mu_{1}$ displayed the sharpest changes in value while the parameters $\mu_{2}, \kappa$, and $\gamma$ changed more gradually. Not surprisingly, the values of $\mu_{2}$ are an order of magnitude larger than $\mu_{1}$ reflecting the difference in stiffness between the collagen fibers and matrix. The values of $\kappa$ are approximately 0.5 in the center indicating that the tissue as a whole is only moderately anisotropic. The non-uniform distribution of $\kappa$ suggests that there are regional differences in the fiber organization. In Fig. 5e, the angle $\theta$ that defines the primary orientation of the collagen fibers is plotted. Note that $\theta$ is defined locally relative to the local basis vector $\mathbf{G}_{1}$ which is parallel to the horizontal meshlines. Keep in mind that when the value of $\kappa$ is approximately 0.5 , the fiber orientation $\theta$ is irrelevant since there is no preferred fiber direction. The algorithmic mean and standard deviation for each of the material parameters are recorded in Table 1.

\subsection{Forward Validation}

Using the pointwise material properties excellent agreement was achieved between the forward finite element prediction and the experimental results. In Fig. 6, the predicted and reconstructed geometries at four pressures are presented. While there are visible deviations at low pressure (Fig. 6a), the configurations at higher

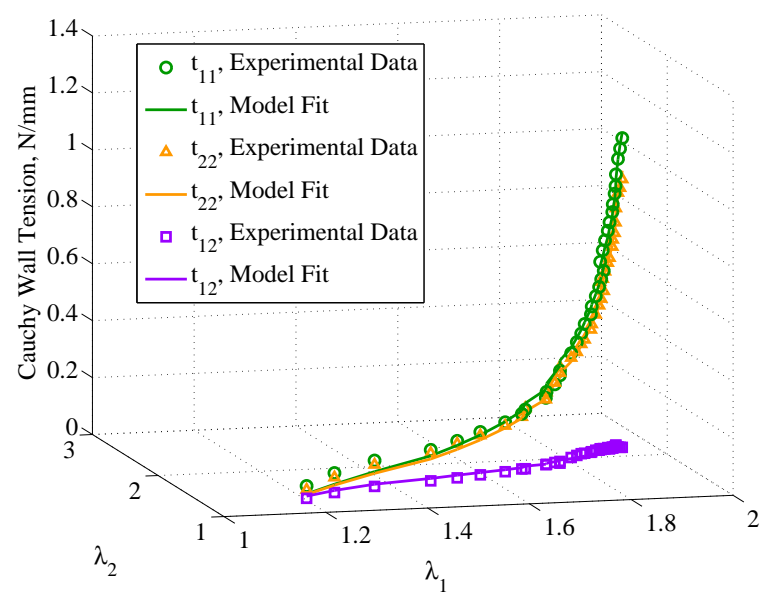

(a)

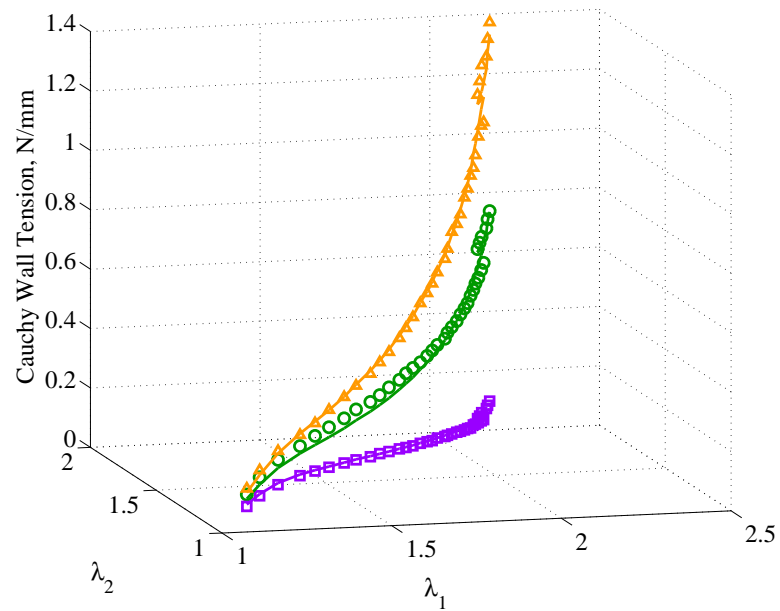

(b)

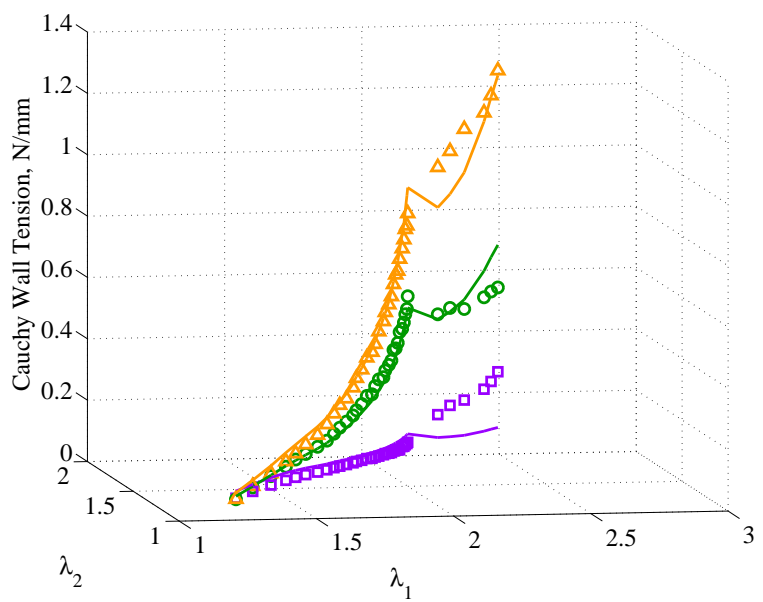

(c)

Fig. 4 Local wall tension versus stretch curves at three different locations. Note that the curves have been rotated into their principal strain state to facilitate plotting. 


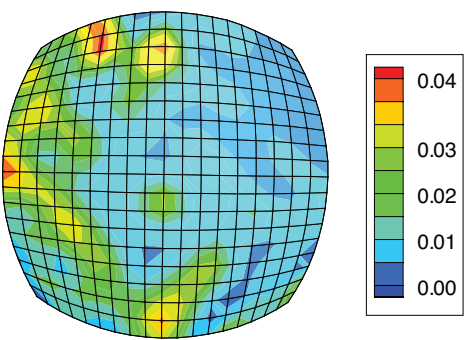

(a) $\mu_{1}(\mathrm{~N} / \mathrm{mm})$

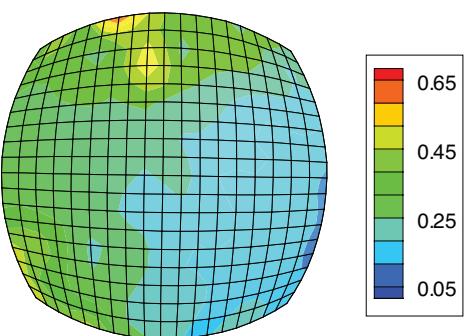

(b) $\mu_{2}(\mathrm{~N} / \mathrm{mm})$

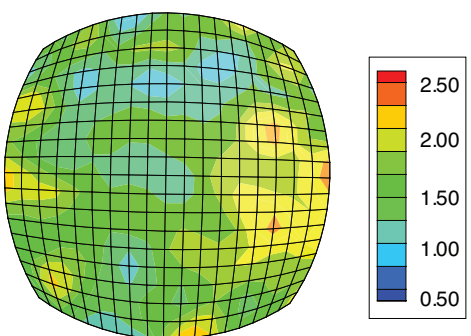

(c) $\gamma$

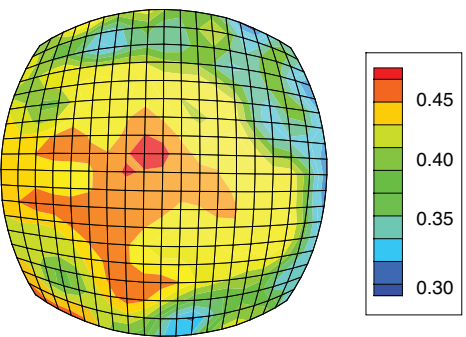

(d) $\kappa$

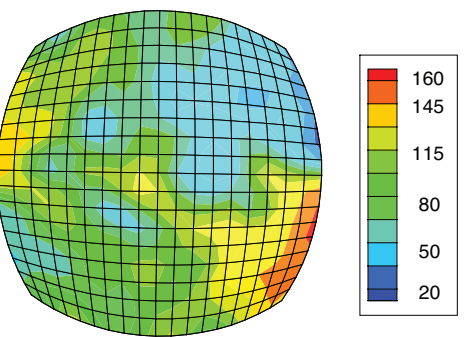

(e) $\theta$ (deg)

Fig. 5 Distribution of the identified material parameters over the ATAA.

Table 2 Comparison of forward finite element predictions using pointwise material properties with the experimental measurements

\begin{tabular}{|c|c|c|c|c|c|c|}
\hline \multirow[t]{2}{*}{ Pressure (kPa) } & \multicolumn{2}{|c|}{ Displacement } & \multicolumn{2}{|c|}{ Wall Tension } & \multicolumn{2}{|c|}{ Strain } \\
\hline & Max. (mm) & Error (\%) & $\operatorname{Max} .(\mathrm{N} / \mathrm{mm})$ & Error (\%) & Max. & Error (\%) \\
\hline 15 & 7.15 & $1.94 \pm 1.68$ & 0.21 & $8.25 \pm 6.54$ & 0.39 & $7.99 \pm 6.33$ \\
\hline 30 & 9.63 & $0.42 \pm 0.42$ & 0.39 & $3.92 \pm 3.35$ & 0.61 & $2.67 \pm 2.50$ \\
\hline 45 & 10.75 & $0.27 \pm 0.25$ & 0.61 & $3.30 \pm 2.84$ & 0.71 & $1.50 \pm 1.44$ \\
\hline 60 & 11.49 & $0.13 \pm 0.14$ & 0.84 & $2.78 \pm 2.45$ & 0.77 & $0.95 \pm 1.00$ \\
\hline 70 & 12.10 & $0.10 \pm 0.11$ & 1.08 & $2.18 \pm 2.00$ & 0.81 & $0.73 \pm 0.85$ \\
\hline 90 & 12.62 & $0.09 \pm 0.11$ & 1.33 & $1.69 \pm 1.68$ & 0.85 & $0.69 \pm 0.85$ \\
\hline 105 & 13.15 & $0.12 \pm 0.14$ & 1.59 & $1.61 \pm 1.73$ & 0.88 & $0.79 \pm 1.08$ \\
\hline 117 & 14.11 & $0.11 \pm 0.17$ & 1.86 & $2.22 \pm 1.95$ & 0.94 & $0.91 \pm 1.45$ \\
\hline
\end{tabular}

Table 3 Comparison of forward finite element predictions using elementwise material properties with the experimental measurements

\begin{tabular}{|c|c|c|c|c|c|c|}
\hline \multirow[t]{2}{*}{ Pressure $(\mathrm{kPa})$} & \multicolumn{2}{|c|}{ Displacement } & \multicolumn{2}{|c|}{ Wall Tension } & \multicolumn{2}{|c|}{ Strain } \\
\hline & $\operatorname{Max} .(\mathrm{mm})$ & Error (\%) & $\operatorname{Max} .(\mathrm{N} / \mathrm{mm})$ & Error (\%) & Max. & Error $(\%)$ \\
\hline 15 & 7.14 & $2.41 \pm 2.68$ & 0.21 & $9.69 \pm 10.27$ & 0.39 & $9.30 \pm 10.06$ \\
\hline 30 & 9.63 & $1.89 \pm 2.04$ & 0.39 & $7.52 \pm 7.44$ & 0.61 & $6.30 \pm 6.74$ \\
\hline 45 & 10.75 & $1.03 \pm 1.09$ & 0.61 & $6.14 \pm 5.96$ & 0.71 & $4.58 \pm 5.29$ \\
\hline 60 & 11.49 & $0.51 \pm 0.59$ & 0.84 & $5.43 \pm 5.62$ & 0.77 & $3.85 \pm 4.93$ \\
\hline 70 & 12.10 & $0.48 \pm 0.59$ & 1.08 & $5.11 \pm 5.83$ & 0.81 & $3.86 \pm 4.97$ \\
\hline 90 & 12.62 & $0.67 \pm 0.77$ & 1.33 & $5.02 \pm 6.02$ & 0.85 & $4.17 \pm 5.19$ \\
\hline 105 & 13.15 & $0.85 \pm 0.95$ & 1.59 & $5.56 \pm 6.34$ & 0.88 & $4.61 \pm 5.55$ \\
\hline 117 & 14.11 & $0.85 \pm 0.93$ & 1.86 & $5.49 \pm 5.70$ & 0.94 & $4.72 \pm 5.72$ \\
\hline
\end{tabular}




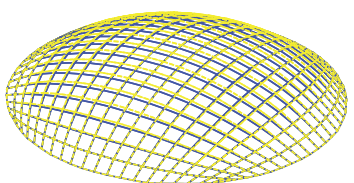

(a) $15 \mathrm{kPa}$

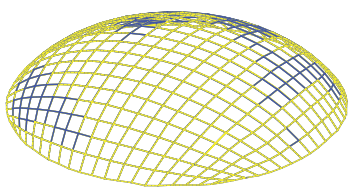

(c) $75 \mathrm{kPa}$

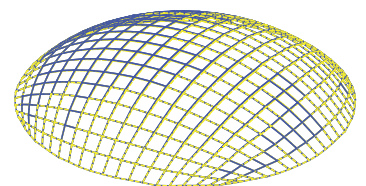

(b) $30 \mathrm{kPa}$

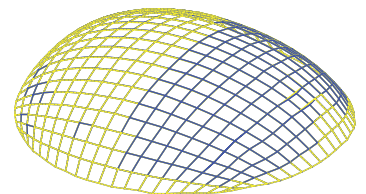

(d) $117 \mathrm{kPa}$
Fig. 6 Comparison of the geometry constructed from the DIC point clouds (blue) and those predicted from the forward finite element analysis using the pointwise material properties (yellow) at (a) $15 \mathrm{kPa}$, (b) $30 \mathrm{kPa}$, (c) $75 \mathrm{kPa}$, and (d) 117 $\mathrm{kPa}$. Note that the geometry is almost a perfect match leading the blue and yellow lines to overlap.

pressures are indistinguishable (Fig. 6b-d). The predicted magnitude of the wall tension and strain were also very similar to their corresponding experimental results, as illustrated in Fig. 7. The quality of the forward prediction is summarized in Table 2 where the average percent error for the wall tension, strain, and displacement are presented at 8 pressures. The highest errors between the forward predictions and experimental data occur at low pressures. The errors then rapidly decline, stabilizing at approximately $60 \mathrm{kPa}$ reflecting the good agreement seen in Figs. 6 and 7. As expected, the smallest errors were found in the displacement predictions. These errors in the displacement then lead to the larger errors observed in the wall tension and strain.

Similar results were found using elementwise material properties as shown in Table 3. Although the errors in displacement, wall tension, and strain are elevated compared to the pointwise case, the mean error for displacement, wall tension, and strain were all below $6 \%$ when the error stabilized. The small magnitude of the errors confirm that the material property distributions were accurately identified.

When the homogeneous material properties given in Table 1 were used, the forward simulation produced large displacement errors, which in turn, generated large errors in the strain and wall tension. The average displacement error for the homogeneous case was approximately $10 \%$ when the error stabilized and the resulting average strain and wall tension errors were on the order of $20 \%$. The large errors that result from assuming homogeneous material properties suggest that the spatial variation in the material parameters must be accounted for in order to accurately replicate the behavior of the ATAA.

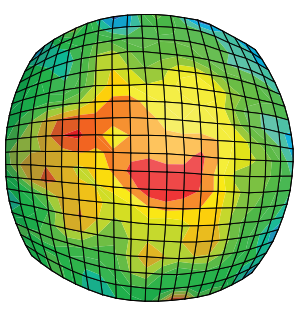

(a) Inverse wall tension

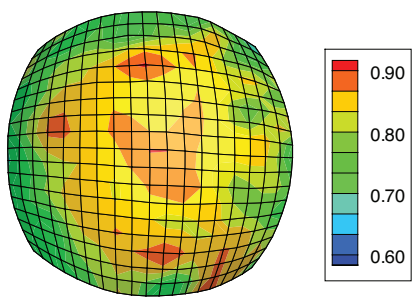

(c) DIC strain

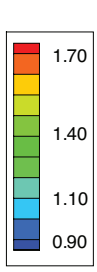

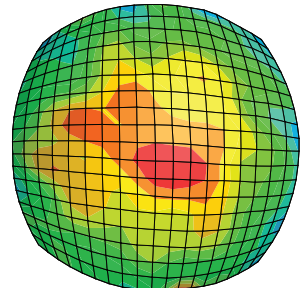

(b) Predicted wall tension

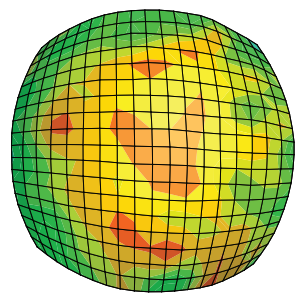

(d) Predicted strain
Fig. 7 Comparison of the magnitude of the $(a, b)$ wall tension $(\mathrm{N} / \mathrm{mm})$ and $(\mathrm{c}, \mathrm{d})$ Green-Lagrange strain from $(\mathrm{a}, \mathrm{c})$ the experimental data and $(b, d)$ the forward finite element prediction at a pressure of $117 \mathrm{kPa}$. Note that the red ellipse in the top center of (c) is the region where rupture initiated.

\section{Discussion}

In this manuscript, we have presented a novel method to identify the pointwise heterogeneous distribution of material properties in soft tissues. The feasibility of the method was demonstrated using a human ATAA. However, in practice, this method could be used to characterize a variety of thin soft tissues such as skin, aortic valves, or the bladder. By combining DIC, bulge inflation testing, and inverse membrane analysis we are able to obtain the stress-strain response locally, at every Gauss point. Using this local stress-strain data the elastic properties were identified. Specifically for the ATAA a modified form of the GOH model was fitted to the local stress-strain curves to identify the heterogeneous distribution of the material properties. A forward validation was also performed to show that a heterogeneous distribution of the material properties must be used to accurately describe the response of the ATAA.

In previous studies using bulge inflation tests, the membrane stresses were frequently identified using the Laplace equation which requires that the membrane is axisymmetric. Investigators assumed that the tissue deformed into either a spherical cap (Mohan and Melvin, 1983) or an elliptical cap (Coudrillier et al., 2012; Marra et al., 2006) so that the Laplace equation could be used. However, during our bulge inflation tests, the ATAA did not maintain an axisymmetric shape but, instead, deformed into a prolate dome without any pronounced symmetry (Fig. 6d). For membranes with no marked 
symmetry, the Laplace equations cannot provide accurate estimations of the stress and the numerical approach presented in this manuscript is necessary.

The protocol presented, in particular the inverse membrane analysis, is quite sensitive to the quality of the displacement data (Zhao et al., 2009). The accuracy of the stress reconstruction depends heavily on local changes in the surface curvature which are calculated from the DIC displacement data. Numerical derivatives are performed on the displacement data to calculate the strain so, any noise in the displacement data is transferred to and amplified in the strain data. When the surface is non-smooth, having small local undulations, the accuracy of the stress and strain reconstruction is also compromised. To overcome these possible accuracy issues, we have employed a stereoscopic DIC system where we have estimated that the displacement measurements are accurate to $30 \mu \mathrm{m}$ (Eq. 1) and second order NURBS elements. The NURBS approach uses isogeometric analysis and results in an intrinsically smooth method to describe the geometry (Hughes et al., 2005), which, in turn, produces smooth stress and strain data. In Fig. 2, the higher distance deviations in the geometric reconstruction (Eq. 4) at pressures below $30 \mathrm{kPa}$ are believed occur due to a non-smooth surface geometry. As the ATAA is inflated, the specimen surface becomes smooth. Reflecting this change in the geometry the mean distance deviation gradually declines and stabilizes around $30 \mathrm{kPa}$. The results of the forward validation (Figs. 6 - 7) confirm that the displacement, strain, and stress were correctly identified using our protocol.

In general the quality of the fits performed using the GOH model were very good but at the low pressures the fits were sometimes poor as seen in Fig. 4. Several factors contributed to the decreased accuracy at low pressures. First, the assumption that the configuration at $3 \mathrm{kPa}$ is stress-free is debatable. The flattening of the ATAA sample from its cylindrical state and the inversion so that the lumen faced out could conceivably lead the assumed reference state to be stressed. Second, by defining the objective function as the sum of the squares difference between the stresses computed from the inverse simulation and the model stresses automatically assigns equal weight to each state, in effect skewing the fits toward the high stress terms. Consequently poor fits at low values of stress and strain do not significantly change the overall value of the objective function. The introduction of a non-uniformly weighted objective function could improve the fits by assigning higher weights to stress-strain points at low pressures.

The material properties identified for the ATAA are highly heterogeneous as evidenced by Fig. 5 . The values of the parameters $\mu_{1}, \mu_{2}, \gamma$, and $\kappa$ ranged from 0.002 $0.04 \mathrm{~N} / \mathrm{mm}, 0.15-0.65 \mathrm{~N} / \mathrm{mm}, 1.00-2.50$, and $0.30-0.46$, respectively. The map of the dispersion parameter, $\kappa$, in Fig. 5d suggests that the ATAA is regionally anisotropic. This finding may explain the conflicting reports in the literature on the whether the thoracic aorta shows directional dependence (Choudhury et al., 2009; Okamoto et al., 2002; Pham et al., 2013; Zhou and Fung, 1997). Figure 5 d reveals that the degree of anisotropy observed is location dependent. This observation could be confirmed by studying of the microstructural organization of the ATAA using histology to independently quantify the values of the structural parameters $\kappa$ and $\theta$.

To show that it is necessary to consider the heterogeneous distribution of the mechanical properties, we performed three different forward finite element simulations using pointwise, elementwise, and homogeneous material properties. In Tables 2 - 3, the errors in displacement, wall tension, and strain for the pointwise and elementwise cases are given. It is clear that homogenization leads to increased errors in the forward finite element simulations. While the errors in the final stage for the elementwise case were 5 times greater than for the pointwise case, the mean errors for displacement, strain, and wall tension were only $0.84 \pm 0.93 \%$, $4.72 \pm 5.72 \%$, and $5.49 \pm 5.70 \%$ respectively. Note that in this case, the use of elementwise properties still requires that the heterogeneities of the material parameters are known at a spatial resolution of $1 \mathrm{~mm}$. For the homogeneous case the mean displacement error by the final stage had only decreased to $7.49 \%$. Large displacement errors $(>5 \%)$ compromise the stress calculations as discussed earlier. Our results suggest that assumption of homogeneity for the ATAA may inhibit researchers' ability to accurately recreate physiologic stresses and strains in finite element simulations.

Although the primary aim of this method is to identify the local elastic response of soft tissues, it is also an apt method for the identification of rupture properties. For our study case of the ATAA, the most commonly used method to estimate the rupture stress is uniaxial tensile testing (Iliopoulos et al., 2009; Sokolis et al., 2012; Vorp et al., 2003). Yet it is well known that the in vivo loading experienced by arterial tissues is much better approximated by biaxial tests (Marra et al., 2006). By design, our approach can easily identify the rupture stress in a non-uniform two-dimensional stress environment, and therefore, the rupture stresses calculated are believed to be more accurate than those identified from uniaxial tests. In addition, our method leaves open the possibility of evaluating the mode of rupture by characterizing how the initial tear propagates in the tissue. 
There are, however, several limitations to our method. First, this protocol is only valid for membranes. From the standpoint of mechanics, a membrane is a solid structure where the thickness is small compared to the other dimensions and the structure's resistance to bending is small. At low pressures $(\mathrm{p}<20 \mathrm{kPa})$, the membrane condition may be compromised due to a relatively small length to thickness ratio when the specimen is only slightly inflated. It is also possible that the bending effects in the ATAA may not be negligible at low pressures where bending is dominant mode of deformation. The nonlinear elastic behavior of the ATAA may amplify the role bending plays in the stress-strain response. Indeed, this may be one of the causes of the large errors that were found in the forward validation at low pressures. Second, only a limited variety of deformations can be produced using a bulge inflation test. For a bulge inflation test one does not prescribe a biaxial deformation or stress state instead, a pressure is specified and the resulting deformation recorded. Therefore it is unclear if the parameters from the bulge inflation test can be used to predict the response of the ATAA in uniaxial tension, for example. Finally, we only considered three types of material property distributions: pointwise, elementwise, and homogeneous. To truly identify the spatial resolution necessary to accurately describe the heterogeneous properties of the ATAA other levels of refinement should be considered. Despite these limitations, our approach provides a novel method that can characterize the complex heterogeneity observed in soft tissues.

In conclusion, we have developed a protocol for characterizing the heterogeneous mechanical properties of thin planar tissues. The true strength of this combined experimental and computational approach is that the method is unaffected by the complexity of the heterogeneity or the nonlinear elastic behavior of the sample. Although the method was applied to the ATAA in the feasibility study, the method could be widely applied to a variety of planar soft tissues. In the future, we plan to conduct a large population study on the ATAA. This study would combine our method with local structural analysis such as histology or multi-photon microscopy to relate the microstructural organization of the tissue to the distribution of the mechanical properties. The additional structural data is necessary to move from merely measuring the heterogeneous nature of the material properties to uncovering the links that exist between the measured properties and the underlying microstructure. We also plan to use the heterogeneous properties from the population study to quantify the influence that material heterogeneity has on the predicted peak wall stress in patient specific finite element models. Furthermore, this method could be transferred to a clinical setting in the future to estimate the material properties of soft tissues in vivo. When coupled with an appropriate imaging modality (e.g. 4D MRI) the evolution of the material properties as the ATAA enlarges could be measured on a patient-to-patient basis as a part of the surveillance routine.

Acknowledgements This work was supported in part by The University of Iowa Mathematical and Physical Sciences Funding Program. Dr. Davis was supported by the Whitaker International Scholars Program. The authors thank Dr. Aaron Romo for his assistance with the experimental protocol.

\section{References}

Belytschko T, Liu WK, Organ D, Fleming M, Krysl P (1996) Meshless methods: An overview and recent developments. Comput Methods Appl Mech Eng 139: $3-47$.

Choudhury N, Bouchot O, Rouleau L, Tremblay D, Cartier R, Butany J, Mongrain R, Leask RL (2009) Local mechanical and structural properties of healthy and diseased human ascending aorta tissue. Cardiovasc Pathol 18(2):83-91. doi:10.1016/j.carpath.2008.01.001

Coudrillier B, Tian J, Alexander S, Myers KM, Quigley HA, Nguyen TD (2012) Biomechanics of the human posterior sclera: Age and glaucoma related changes measured using inflation testing. Invest Ophthalmol Vis Sci, 53(4):1714-28 doi:10.1167/iovs.118009.

Gasser TC, Ogden RW, Holzapfel GA (2006) Hyperelastic modelling of arterial layers with distributed collagen fibre orientations. $J R$ Soc Interface, 3(6): 15-35. doi:10.1098/rsif.2005.0073

Genovese K, Casaletto L, Humphrey JD, Lu J (2014) Digital image correlation-based point-wise inverse characterization of heterogeneous material properties of gallbladder in vitro. Proc $R$ Soc A, 470:20140152. doi:10.1098/rspa.2014.0152

Green AE, Adkins JE (1970) Large elastic deformations. Clarendon Press Oxford

Haskett D, Johnson G, Zhou A, Utzinger U, Vande Geest JP (2010) Microstructural and biomechanical alterations of the human aorta as a function of age and location. Biomech Model Mechanobiol, 9 (6):725-36. doi:10.1007/s10237-010-0209-7

Hughes TJR, Cottrell JA, Bazilevs Y (2005) Isogeometric analysis: CAD, finite elements, NURBS, exact geometry and mesh refinement. Comput Methods Appl Mech Eng, 194(39):4135-4195. 
Humphrey JD (2003) Review Paper: Continuum biomechanics of soft biological tissues. Proc $R$ Soc A , 459 (2029):3-46. doi:10.1098/rspa.2002.1060

Humphrey JD, Milewicz DM, Tellides G, Schwartz MA (2014) Dysfunctional mechanosensing in aneurysms. Sci, 344(6183):477-479. doi:10.1126/science.1253026

Iliopoulos DC, Deveja RP, Kritharis EP, Perrea D, Sionis GD, Toutouzas K, Stefanadis C, Sokolis DP (2009) Regional and directional variations in the mechanical properties of ascending thoracic aortic aneurysms. Med Eng Phys, 31:1-9. doi:10.1016/j.medengphy.2008.03.002

Kroon M, Holzapfel GA (2008) A new constitutive model for multi-layered collagenous tissues. $J$ Biomech, 41(12):2766-71. doi:10.1016/j.jbiomech.2008.05.033

Lu J, XF Zhao (2009) Pointwise identification of elastic properties in nonlinear hyperelastic membranes-Part I: Theoretical and computational developments. $J$ Appl Mech, 76(6):061013. doi:10.1115/1.3130805

Lu J, Zhou XL, Raghavan ML (2007) Computational method for inverse elastostatics for anisotropic hyperelastic solids. Int J Numer Methods Eng, 69:12391261.

Lu J, Zhou X, Raghavan ML (2008) Inverse method of stress analysis for cerebral aneurysms. Biomech Model Mechanobiol, 7(6):47786. doi:10.1007/s10237-007-0110-1

Lu J (2011) Isogeometric contact analysis: Geometric basis and formulation for frictionless contact. Comput Methods Appl Mech Eng, 200(5-8):726-741. doi:10.1016/j.cma.2010.10.001

Lu J, Hu S, Raghavan ML (2013) A shell-based inverse approach of stress analysis in intracranial aneurysms. Ann Biomed Eng, 41(7):1505-1515.

Marra SP, Kennedy FE, Kinkaid JN, Fillinger MF (2006) Elastic and rupture properties of porcine aortic tissue measured using inflation testing. Cardiovasc Eng, 6(4):123-31. doi:10.1007/s10558-006-90215

Miller K, Lu J (2013). On the prospect of patientspecific biomechanics without patient-specific properties of tissues. J Mech Behav Biomed Mater, 27: 154-166.

Mohan DH, Melvin JW (1983). Failure properties of passive human aortic tissue. II- Biaxial tension tests. $J$ Biomech, 16(1):31-44.

Myers KM, Coudrillier B, Boyce BL, Nguyen TD (2010) The inflation response of the posterior bovine sclera. Acta Biomater, 6(11):4327-35. doi:10.1016/j.actbio.2010.06.007

Ní Annaidh A, Bruyère K, Destrade M, Gilchrist MD, Otténio M (2012) Characterization of the anisotropic mechanical properties of excised human skin. J Mech Behav Biomed Mater, 5(1):139-48. doi:10.1016/j.jmbbm.2011.08.016

Okamoto RJ, Wagenseil JE, DeLong WR, Peterson SJ, Kouchoukos NT, Sundt III TM (2002) Mechanical properties of dilated human ascending aorta. Ann Biomedial Eng, 30:624-635. doi:10.1114/1.1484220

Pham T, Martin C, Elefteriades JA, Sun W (2013) Biomechanical characterization of ascending aortic aneurysm with concomitant bicuspid aortic valve and bovine aortic arch. Acta Biomater, 9:7927-7936. doi:10.1016/j.actbio.2013.04.021

Reu P(2014). The art and application of DIC: Calibration sanity checks. Exp Techniques, 38(2):1-2, doi:10.1111/ext.12077

Romo A, Badel P, Duprey A, Favre JP, Avril S (2014) In vitro analysis of localized aneurysm rupture. $J$ Biomech, 47(3):607-616.

Sokolis DP, Kritharis EP, Iliopoulos DC (2012) Effect of layer heterogeneity on the biomechanical properties of ascending thoracic aortic aneurysms. Med Biol Eng Comput, 50:1227-1237. doi:10.1007/s11517-0120949-x

Tonge TK, Atlan LS, Voo LM, Nguyen TD (2013) Full-field bulge test for planar anisotropic tissues: Part I-Experimental methods applied to human skin tissue. Acta Biomater, 9(4):5913-25. doi:10.1016/j.actbio.2012.11.035

Vorp DA (2007) Biomechanics of abdominal aortic aneurysm. $J$ Biomech, 40:1887-1902. doi:10.1016/j.jbiomech.2006.09.003

Vorp DA, Shiro BJ, Ehrlich MP, Juvonen TS, Ergin MA, Griffith BP (2003) Effect of aneurysm on the tensile strength and biomechanical behavior of the ascending thoracic aorta. Ann Thorac Surg, 75: 1210-1214.

Wilson JS, Baek S, Humphrey JD (2012) Importance of initial aortic properties on the evolving regional anisotropy, stiffness and wall thickness of human abdominal aortic aneurysms. J R Soc Interface, 9:20472058. doi:10.1098/rsif.2012.0097

Zhao X, Chen X, Lu J (2009) Pointwise identification of elastic properties in nonlinear hyperelastic membranes - Part II: Experimental validation. J Appl Mech, 76(6):061014. doi:10.1115/1.3130810

Zhao X, Raghavan ML, Lu J (2011) Characterizing heterogeneous properties of cerebral aneurysms with unknown stress-free geometry: A precursor to in vivo identification. J Biomech Eng-T ASME, 133 (2):051008.

Zhao X, Raghavan ML, Lu J (2011) Identifying heterogeneous anisotropic properties in cerebral aneurysms: A pointwise approach. Biomech Model Mechanobiol, 
10(2):177-189.

Zhou J, Fung YC (1997) The degree of nonlinearity and anisotropy of blood vessel elasticity. Proc Natl Acad Sci, 94(26):14255-14260. doi:10.1073/pnas.94.26.14255 